\title{
Effect of glyceryl trinitrate on echocardiographic left ventricular dimensions during exercise in the upright position
}

\author{
ROBERT E. GOLDSTEIN ${ }^{1}$, E. DAVID BENNETT, AND GRAHAM L. LEECH
}

From the Cardiac Department and the Medical Department, St. George's Hospital, London

SUMMARY Changes in left ventricular size may be of importance in the development of angina and in its amelioration by glyceryl trinitrate, but left ventricular dimensions have not been measured during exercise in the upright position, the circumstance in which angina most often occurs. To assess changes in left ventricular end-systolic and end-diastolic dimensions, echocardiograms were obtained from 5 normal subjects and 6 patients with angina during rest and exercise upright, both before and after glyceryl trinitrate. The end-systolic dimension was considered an index of regional performance rather than an estimate of overall left ventricular behaviour. During exercise the end-diastolic dimension rose both in the normal subjects and in the patients. The end-systolic dimension fell progressively with exercise in the normal subjects but changed inconsistently in the patients. Glyceryl trinitrate lowered the end-diastolic dimension during exercise below comparable pretreatment values in both groups; the glyceryl trinitrate-induced decrease in end-diastolic dimension in the patients diminished at higher exercise levels. In all normal subjects and in 4 of the 6 patients with normal systolic shortening (= enddiastolic-end-systolic dimension) glyceryl trinitrate caused relatively small changes in the end-systolic dimension and systolic shortening on exercise. In contrast, in the 2 patients with hypokinetic ventricles glyceryl trinitrate conspicuously decreased the end-systolic dimension and increased systolic shortening.

Thus, during exercise, in the normal subjects and in the patients with normally functioning left ventricles on echocardiography, glyceryl trinitrate caused a modest fall in the end-diastolic dimension and little change in the end-systolic dimension, but in patients with ventricular hypokinesia it greatly improved regional contractile performance. Alleviation of ischaemic hypokinesia during exercise may be an important part of the benefit of glyceryl trinitrate.

Much is known about left ventricular dimensions during exercise supine (Sonnenblick et al., 1965; Sharma et al., 1976; Stein et al., 1976; Borer et al., 1978) but far less about the changes which occur when exercise is performed in an upright position. By favouring venous pooling within dependent portions of the body, the upright posture has been shown to cause a substantial reduction in left ventricular cavity dimensions in normal subjects at rest (DeMaria et al., 1974; Redwood et al., 1974). The extent to which this potential for venous pooling influences left ventricular dimensions during exercise when upright, however, has not been

${ }^{1}$ Dr Goldstein was a John Simon Guggenheim Memorial Fellow

Received for publication 22 August 1978 measured. Such postural influence may be particularly important when assessing the action of glyceryl trinitrate (GTN). Increased venous pooling is presumably a major factor causing decrease in left ventricular size when GTN is administered to resting upright subjects (DeMaria et al., 1974; Redwood et al., 1974) and though during exercise upright this mechanism is thought to play a significant part in the antianginal action of GTN, data documenting such an effect are lacking. Accordingly, echocardiographic measurements were made during exercise in the upright position both before and after glyceryl trinitrate in normal subjects and in patients with angina pectoris. Though echocardiographic results are not necessarily representative of dimensional changes in portions of the left ventricle not traversed by the ultrasound beam, particularly when systolic measurements are made 
in patients with coronary artery disease, data during systole may be useful in assessing changes in regional performance.

\section{Methods}

\section{SUBJECTS}

Technically satisfactory echocardiograms were obtained during exercise in 5 normal male subjects, aged 23 to 48 years, in whom the history, physical examination, electrocardiogram, and echocardiogram disclosed no evidence of cardiovascular disease. Four other normal subjects were excluded from the study because satisfactory echocardiograms could not be obtained during exercise.

Seven male patients, aged 36 to 61 years, had satisfactory echocardiograms during exercise. These subjects had been selected at random from an outpatient population with symptomatic coronary artery disease. Each had had typical stable angina pectoris for a varying duration (range 6 months to 12 years). Documented myocardial infarction (with persistent $Q$ waves) had occurred several years previously in 2 patients. Electrocardiograms showed nonspecific $T$ wave abnormalities at rest in 1 additional patient and significant ST segment depression during exercise in 2 others. Clinical examination showed no evidence of any other disease. No patient had an arrhythmia or overt congestive cardiac failure. Cardiac catheterisation was not performed in any subject. Four further patients with angina were excluded from the study because of technically unsatisfactory echocardiograms during exercise.

All subjects were fully acquainted with the purpose and procedures of the study and informed consent was obtained from each before participation.

\section{STUDY PLAN}

Echocardiograms were obtained at rest supine, at rest upright (subjects seated on a bicycle ergometer with their feet on the pedals), during the last 30 seconds of each 3-minute exercise level, and when a limiting symptom, such as angina appeared. Echocardiograms were recorded using an Aerotech Gamma transducer $(2.25 \mathrm{MHz}, 1.3 \mathrm{~cm}$ diameter, $10 \mathrm{~cm}$ focus) connected to an Ekoline 20 ultrasound unit. A Cambridge recorder (model 72112) transferred the resultant signal to light-sensitive paper moving at a speed of $50 \mathrm{~mm} / \mathrm{s}$. A conventional Tscanning technique was employed to ensure that measurements were made at a consistent site (Henry et al., 1973). Satisfactory echocardiograms could usually be recorded during exercise on our upright bicycle ergometer (Godart) if subjects leaned forward. The operator held the transducer at the junction of the fourth or fifth intercostal space and the left sternal border, adjusting the transducer angle as necessary during exercise to maintain the desired image on a monitoring oscilloscope. In addition, an electrocardiographic lead (CM5) was monitored and systolic blood pressure was measured (using a pneumatic arm cuff) at rest and during exercise.

Normal subjects exercised for 3 minutes at each of 4 escalating work loads $(40,80,120$, and 160 Watts) without pausing. Using a procedure previously established (Redwood et al., 1971) the work load for the patients with coronary disease, however, was increased by 20 Watts every 3 minutes, with the initial work load chosen individually so that angina developed in each subject when unprotected by GTN between the 4th and 7th minute of exercise, and this value was identified at practice sessions before the day of study. The work load at the point that angina occurred during the study (no GTN having been given) varied from 60 to 140 Watts, the patients' heart rates then ranging from 108 to 127 beats/min. Exercise was stopped (recordings having been obtained) within 30 seconds of the onset of pain. Unprotected by GTN all patients stopped exercise because of angina; after GTN 3 were halted at higher work loads by fatigue instead. All subjects showed normal rhythm and conduction throughout.

Studies were performed in the postabsorptive state. The 4 patients taking propranolol discontinued this drug at least 24 hours beforehand and no patient had had GTN for at least 12 hours. Each subject exercised first without drugs and then after a placebo sublingually. Finally, all normal subjects and 6 of the 7 patients exercised 3 minutes after sublingual GTN, $0.5 \mathrm{mg}$. Thirty minutes' rest supine was interposed between each period of exercise. (NB: Previous work (Redwood et al., 1971) showed no change in exercise capacity in patients with angina during a third period of exercise unless such exercise was preceded by sublingual trinitrate administration.) The dose of GTN employed was sufficient to raise the heart rate at least 10 beats/min or lower the systolic blood pressure at least $10 \mathrm{mmHg}$ in each subject when measurements were made at rest upright and it produced a rise in exercise capacity in each of the 6 patients with coronary disease (mean increase in duration of exercise $=$ $3.8 \mathrm{~min}$ ).

In 2 of the normal subjects, parts of the procedure were repeated on a subsequent day to evaluate the reproducibility of the measurements.

DATA ANALYSIS

Maximum and minimum values of the distance 


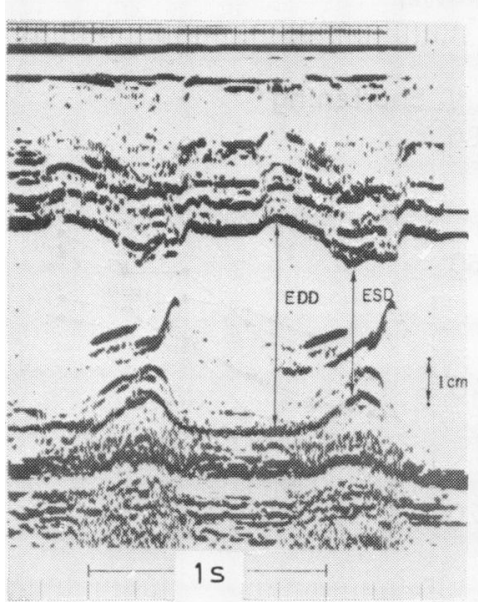

Supıne rest
Exercise echocardiograms

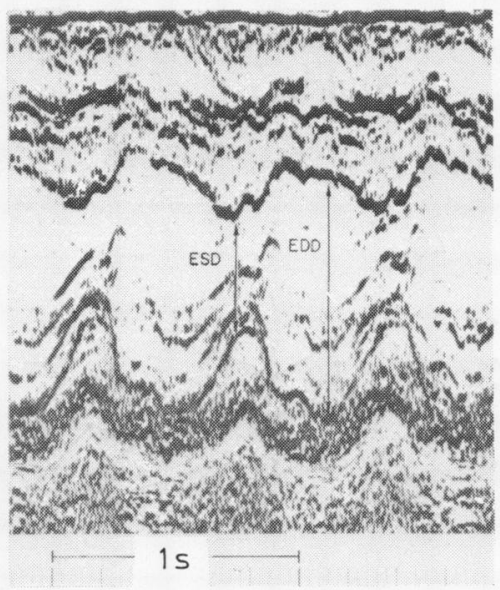

80 watts

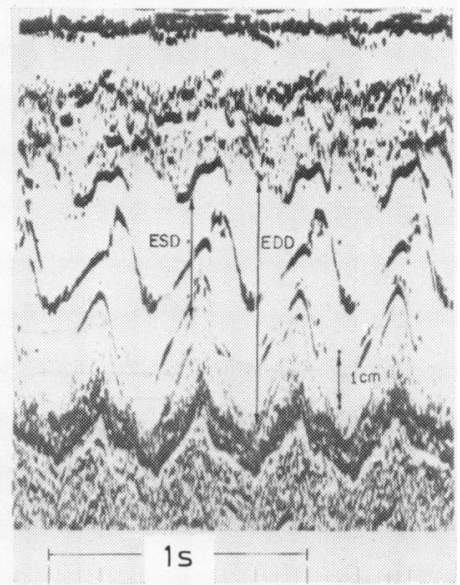

160 watts

Fig. 1 Echocardiograms are shown for one normal subject at rest supine (left), during moderate exercise (centre), and during strenuous exercise (right) on an upright bicycle ergometer. EDD, end-diastolic dimension; ESD, end-systolic dimension. Calibration markers, spaced $1 \mathrm{~cm}$ apart along a vertical axis, are identified in left and right panels.

between left ventricular septal and free wall endocardial surfaces were taken to represent the left ventricular end-diastolic dimension (EDD) and end-systolic dimension (ESD), respectively (Fig. 1). Measurements were taken from the posterior border of the septal echo. The percentage shortening of left ventricular dimension was calculated as $(1-\mathrm{ESD} / \mathrm{EDD}) \times 100$ per cent. Between 5 and 12 complexes (mean 8) were measured and the measurements averaged for each patient at each exercise level. Each resultant data point had a standard deviation averaging $\pm 1.0 \mathrm{~mm}$ and a standard error averaging $\pm 0.34 \mathrm{~mm}$.

Paired comparisons were made using either twotailed Student's $\mathrm{t}$ tests or a two-way analysis of variance.

\section{Results}

\section{REPRODUCIBILITY}

Measurements of left ventricular dimensions during comparable levels of exercise showed no significant alterations in 2 normal subjects studied on 2 different days. Mean changes in 16 determinations $( \pm S D)$ comparing the first and second sets of results were $-1.5 \mathrm{~mm} \pm 3.2$ for the end-diastolic dimension, $0.9 \mathrm{~mm} \pm 2.4$ for the end-systolic dimension, and 0.0 per cent \pm 6.0 for percentage shortening. None of these differences is statistically significant. Similarly, the measurements made during exercise after placebo were indistinguishable from those obtained before it. In the 12 subjects studied mean changes $( \pm S D)$ between the first and second periods of exercise were $0.5 \mathrm{~mm} \pm 1.8$ for the end-diastolic dimension, $0.2 \mathrm{~mm} \pm 2.1$ for the end-systolic dimension, and 0.0 per cent \pm 3.5 for percentage shortening. None of these differences is statistically significant. Equally satisfactory reproducibility was observed in normal subjects and patients alike.

\section{NORMAL SUBJECTS}

The end-diastolic dimension decreased (mean $8.6 \%$, $P<0.05)$ when the upright posture was assumed (Fig. 2, left). The lowest level of exercise, 40 Watts, caused a rise in end-diastolic dimension in all 5 subjects (mean $8.7 \%, \mathrm{P}<0.01$ ) and this tended to reverse the fall associated with the upright posture. At higher exercise levels no consistent pattern was seen. Heart rates and systolic blood pressures before and during exercise are shown in the Table. There was no correlation with changes in enddiastolic dimension. The end-systolic dimension decreased uniformly (mean $14 \%, P<0.01$ ) when supine subjects changed to the upright position (Fig. 2, centre) and decreased further progressively during the course of exercise. At maximal exercise levels the end-systolic dimension was, on average, 25 per cent below values at rest upright $(P<0.01)$. Change from the supine to the upright position was associated with a small rise in percentage shortening $(3 \%, P<0.05)$ (Fig. 2, right). In each subject, mean percentage shortening increased progressively with exercise from 41 per cent at rest upright to 59 per cent during maximal exercise $(P<0.01)$.

The effect of GTN was assessed by examining differences between measurements made after this 

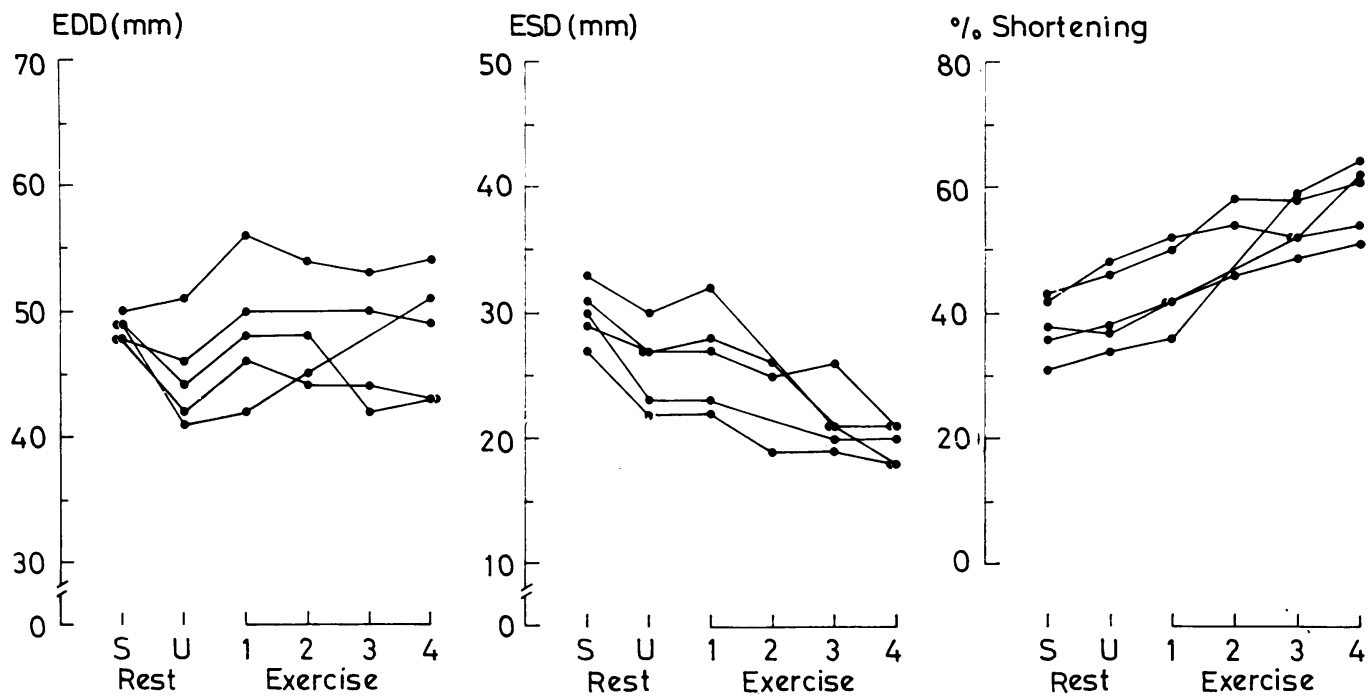

Fig. 2 Values of end-diastolic dimension (left), end-systolic dimension (centre), and percentage systolic shortening $(=(1-E S D / E D D) \times 100$ per cent, right $)$ are shown as connected dots for each normal subject. $S$, rest supine; $U$, rest upright; exercise levels are numbered in order of their performance: 1, $40 \mathrm{Watts}$; 2, 80 Watts; 3, 120 Watts; and 4, 160 Watts.

Table Mean heart rate and blood pressure before and after GTN*

\begin{tabular}{|c|c|c|c|c|c|}
\hline & \multirow[b]{2}{*}{ Rest upright } & \multicolumn{4}{|c|}{ Exercise levels } \\
\hline & & 40 Watts & 80 Watts & 120 Watts & 160 Watts \\
\hline \multicolumn{6}{|l|}{ Normal subjects $(n=5)$} \\
\hline \multicolumn{6}{|l|}{ Heart rate } \\
\hline After placebo & $77 \pm 3$ & $100 \pm 3$ & $114 \pm 6$ & $135 \pm 8$ & $156 \pm 5$ \\
\hline After GTN & $102 \pm 5$ & $111 \pm 6$ & $127 \pm 6$ & $144 \pm 8$ & $163 \pm 6$ \\
\hline \multicolumn{6}{|l|}{ Systolic pressure } \\
\hline After placebo & $122 \pm 8$ & $137 \pm 6$ & $150 \pm 6$ & $168 \pm 4$ & $186 \pm 8$ \\
\hline After GTN & $106 \pm 5$ & $122 \pm 6$ & $137 \pm 11$ & $151 \pm 8$ & $161 \pm 7$ \\
\hline Patients with angina $(n=6)$ & Rest upright & Level 1 & Level 2 & Level 3 & \\
\hline \multicolumn{6}{|l|}{ Heart rate } \\
\hline After placebo & $79 \pm 2$ & $113 \pm 4$ & $124 \pm 4$ & - & \\
\hline After GTN & $90 \pm 2$ & $113 \pm 4$ & $125 \pm 4$ & $136 \pm 4$ & \\
\hline \multicolumn{6}{|l|}{ Systolic pressure } \\
\hline After placebo & $110 \pm 3$ & $138 \pm 8$ & $142 \pm 7$ & - & \\
\hline After GTN & $100 \pm 4$ & $132 \pm 9$ & $136 \pm 9$ & $143 \pm 9$ & \\
\hline
\end{tabular}

* Mean values are shown \pm standard error. Exercise levels for anginal patients are explained in text.

Abbreviations: GTN, glyceryl trinitrate. 
Normal subjects:changes after GTN

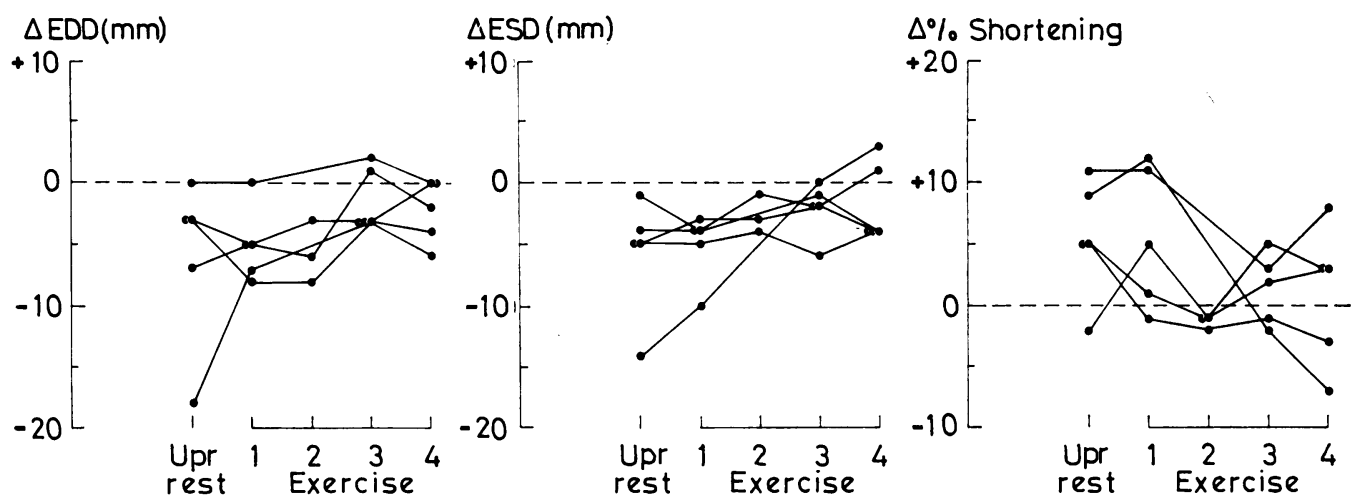

Fig. 3 Changes in end-diastolic dimension (left), end-systolic dimension (centre), and percentage systolic shortening (right) after glyceryl trinitrate (GTN) are shown for each normal subject at rest upright (Upr) and during 4 levels of exercise. Displacement below the zero line (dashes) indicates decrease relative to comparable pre-GTN values.
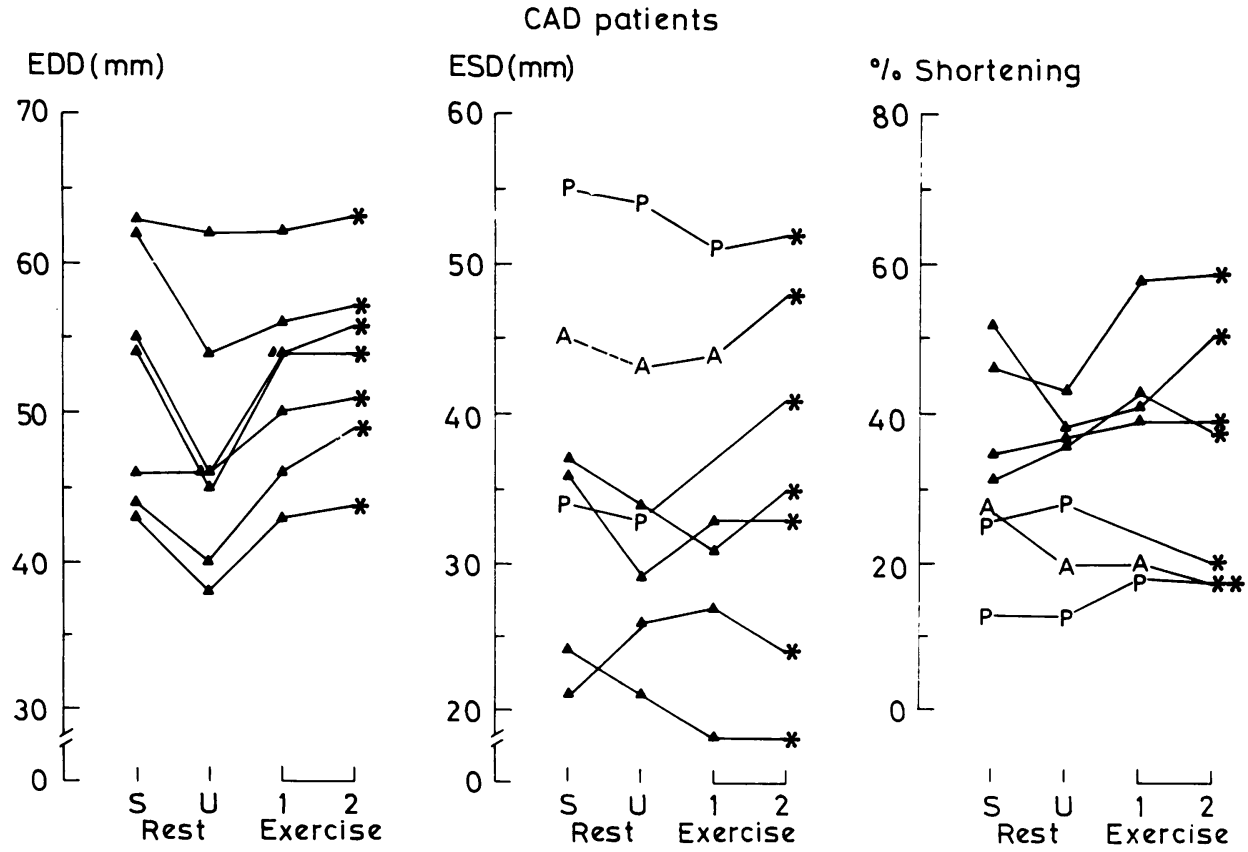

Fig. 4 End-diastolic dimension (left), end-systolic dimension (centre), and percentage systolic shortening (right panel) are shown as connected triangles or letters for each patient with angina. $S$, rest supine; $U$, rest upright; 1 , subanginal exercise level (actual workload chosen individually for each patient); 2, exercise level (20 Watts more intense than level 1) associated with the onset of angina (asterisk) if exercise is not preceded by effective therapy; $P$, patients with posterior hypokinesia; $A$, patient with anterior hypokinesia. $A$ point is omitted from the curve of one patient with posterior hypokinesia because satisfactory measurement of the end-systolic dimension could not be made from the echocardiogram obtained during level 1 exercise. 
drug and those made after placebo (Fig. 3). GTN reduced the end-diastolic dimension by a mean of 16 per cent $(P<0.05)$ in normal subjects at rest upright. The decrease in end-diastolic dimension persisted during the lower two levels of exercise (Fig. 3, left), but diminished at the higher two levels. Thus, the mean decrease was $5 \mathrm{~mm}$ or 10 per cent at 40 Watts $(P<0.05)$, but only $2.4 \mathrm{~mm}$ or 5 per cent (NS) at 160 Watts. The end-systolic dimension was consistently reduced after GTN by a mean of $5 \mathrm{~mm}$ or 19 per cent $(\mathrm{P}<0.02)$ at 40 Watts, and $1.6 \mathrm{~mm}$ or 7 per cent (NS) at 160 Watts (Fig. 3, centre). GTN produced no consistent change in percentage shortening (Fig. 3, right). In no instance did percentage shortening after GTN increase by more than 8 per cent in normal subjects at exercise levels above 40 Watts.

Heart rates and systolic blood pressures during exercise after GTN are given in the Table.

\section{PATIENTS WITH ANGINA}

Patients showed a wider range of left ventricular dimensions than normal subjects; some had an abnormally large end-diastolic dimension (Fig. 4, left) and end-systolic dimension (Fig. 4, middle) when at rest supine. The end-diastolic dimension behaved much as it did in normal subjects: the decrease on the assumption of the upright position (mean $10 \%, \mathrm{P}<0.01$ ) was almost always reversed completely at the lower level of exercise. A small but consistent additional increase in the end-diastolic dimension (mean $1.6 \mathrm{~mm}$ or $3.1 \%, P<0.05$ ) was noted at the higher level of exercise which produced angina. Unlike the normal subjects, the patients did not show a uniform fall in end-systolic dimension and rise in percentage shortening during exercise. On the contrary, end-systolic dimension remained unchanged or increased in 3 of 6 patients and percentage shortening remained virtually unchanged $( \pm 1 \%)$ or decreased during the higher exercise level in 5 of 6 patients. In a seventh patient end-systolic dimension could not be measured during the lower exercise level. Percentage shortening remained below 25 per cent throughout the course of exercise in 3 patients (Fig. 4, right). These individuals were considered to have distinctly hypokinetic segments within the region assessed by echocardiography. Percentage shortening actually decreased below resting values in 2 of these 3 patients; poor septal contraction was responsible in 1 and poor free wall contraction in the other 2.

The effect of GTN was assessed by examining the differences between measurements made after this drug and those made after placebo. When after
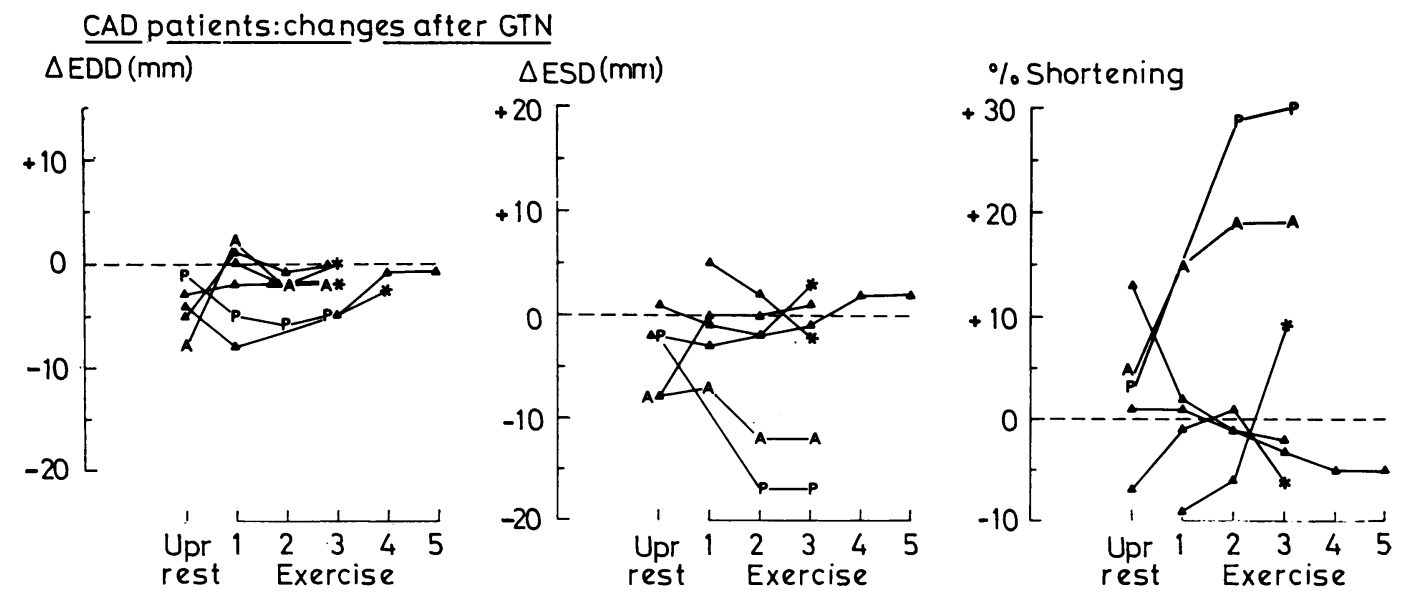

Fig. 5 Changes in end-diastolic dimension (left), end-systolic dimension (centre), and percentage systolic shortening (right) after glyceryl trinitrate (GTN) are shown at rest upright (Upr) and during exercise. Each successive exercise level had a workload 20 Watts greater than the preceding level. Level 1 (same workload as level 1, Fig. 4) was chosen individually as described in text. Patients were able to reach levels 3 to 5 only after GTN. Thus, the value shown at each of these exercise levels represents the difference between measurement after GTN at levels 3, 4, or 5, and the measurement made for the same patient during maximal exercise before GTN (level 2). Termination of post-GTN exercise by angina is denoted by an asterisk; lines ending without an asterisk indicate post-GTN limitation by fatigue. Post-GTN systolic function during exercise was much improved in patients with posterior ( $P$ ) or anterior $(A)$ hypokinesia during pre-GTN exercise. Nevertheless, one of these patients $(A)$ showed very little change in end-diastolic dimension during post-GTN exercise. Two points are omitted from one curve and one point from a second curve in the centre and right panels where the end-systolic dimension could not be measured satisfactorily. 


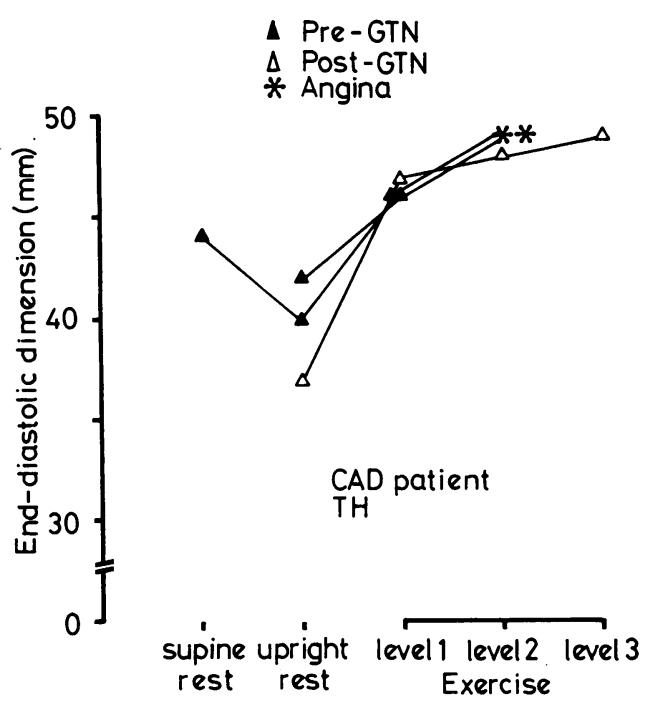

Fig. 6 End-diastolic dimension for one particular patient with angina $(T . H$.$) is shown at rest and during$ exercise at $100 \mathrm{Watts}$ (level 1) and $120 \mathrm{Watts}$ (level 2) before (closed triangles) and after (open triangles) glyceryl trinitrate (GTN) and at 140 Watts (level 3) after GTN. Results during the first exercise level (no prior therapy) are joined to a preceding measurement at rest supine. The second exercise level (post-placebo) yielded very similar values to the first and, like the first, ended with onset of angina (asterisk). The third exercise level (post-GTN) showed a 3-mm reduction in the end-diastolic dimension at rest upright but no reduction greater than $1 \mathrm{~mm}$ during any of the exercise levels. Nevertheless, angina did not appear after GTN, even at $a$ workload 20 Watts greater and at an end-diastolic dimension equal to that at the onset of angina before GTN.

GTN, patients were able to exercise at levels not reached after placebo, measurements at the highest level attained in each case were used for purposes of comparison.

At rest upright the end-diastolic dimension was uniformly decreased (mean $10 \%, \mathrm{P}<0.01$ ) after GTN (Fig. 5, left). Those 2 patients able to exercise longest after GTN showed persistent substantial ( $\geqslant 5 \mathrm{~mm}$ ) GTN-induced decrement in end-diastolic dimension during most of their exercise. In 3 other patients, however, the GTN-induced decrease in end-diastolic dimension seen at upright rest dwindled to $\leqslant 2 \mathrm{~mm}(4 \%)$ or disappeared entirely during the first or second exercise level. In a fourth patient with no rest measurement, GTN-induced decrease in end-diastolic dimension remained small ( $\leqslant 2 \mathrm{~mm}$ ) throughout the course of exercise. Thus, the beneficial effects of GTN were sometimes, not always, accompanied by a substantial decrease in the end-diastolic dimension during exercise. There was no difference in heart rate or systolic pressure distinguishing individuals with substantial GTNinduced decrements in end-diastolic dimension at higher levels of exercise from others who did not show this.

GTN-induced changes in end-systolic dimension and percentage shortening (Fig. 5, middle and right) were even less uniform. In the 4 patients with relatively good systolic function on the echocardiogram, GTN effects were minor and inconsistent, but in 2 of the 3 in whom the ultrasound beam showed severe hypokinesia GTN caused a pronounced, sustained decrease in the end-systolic dimension (maximum 12 and $17 \mathrm{~mm}$ ) and a very large increase in percentage shortening (maximum 19 and $30 \%$ ). The third patient with hypokinesia was not studied after GTN. Thus, GTN improved systolic function during exercise in hypokinetic regions of the left ventricle but had no consistent effect on the ventricles of normal subjects, or on those of patients in whom myocardial contraction on echocardiography had been shown to be relatively normal beforehand.

Heart rates and systolic blood pressures in this group are also given in the Table.

\section{Discussion}

Our findings indicate that satisfactorily reproducible values for the echocardiographic left ventricular end-diastolic and end-systolic dimensions can be obtained during bicycle exercise in the upright position. In the homogeneously contracting hearts of normal subjects it is likely that changes in left ventricular cavity dimensions along the single axis defined by the ultrasound beam are qualitatively (if not quantitatively) similar to changes occurring elsewhere within the left ventricle. Such similarity is unlikely in the less homogeneous contraction often seen in the ventricles of patients with coronary disease, particularly during systole, but even in this group changes in left ventricular cavity dimensions may be valuable in assessing local responses to physiological or pharmacological influences.

\section{LEFT VENTRICULAR DIMENSIONS IN NORMAL SUBJECTS}

Left ventricular end-diastolic and end-systolic dimensions decreased, and percentage shortening rose slightly when the supine normal subjects assumed the upright position (Fig. 2), findings that are in agreement with a previous study on the effect of postural change on echocardiographic dimensions (Redwood et al., 1974). Reversal of the decrease in the end-diastolic dimension occurred at 
the lowest level of exercise, probably because of mobilisation of blood pooled in dependent portions of the body by the pumping action of exercising muscles and increased respiration, and by increased sympathetic venoconstrictor activity (Bevegård and Shepherd, 1965, Freyschuss, 1970), but little further change was noted in most subjects during much more strenuous effort. Stein et al. (1976) reported similar findings in supine subjects. Thus, under ordinary physiological conditions the left ventricular end-diastolic dimension does not appear to increase beyond the value observed at rest when supine. However, progressively more strenuous exercise in the upright position produced correspondingly progressive decreases in the end-systolic dimension and rises in percentage shortening. These data are consistent with evidence from radionuclide cineangiography that the ejection fraction rises progressively in supine normal subjects when they perform increasingly strenuous exercise (Borer et al., 1977). Though caution must be used in accepting our data as representative of dimensional changes occurring throughout the left ventricle, they suggest that when upright normal subjects perform exercise, the concomitant rise in left ventricular output is produced primarily by increases in heart rate and in systolic emptying with little change in left ventricular end-diastolic volume.

GTN decreased the end-diastolic and endsystolic dimensions below corresponding placebo values at rest when upright (Fig. 3), reflecting, in part, the tendency of this drug to exaggerate venous pooling in the upright position. The size of these changes caused by GTN in our seated subjects corresponds more closely to those seen in subjects at $30^{\circ}$ head-up tilt (DeMaria et al., 1974; Redwood et al., 1974) than to those in supine subjects (Williams et al., 1965; Burggraf and Parker, 1974; DeMaria et al., 1974). GTN-induced decreases in left ventricular dimensions diminished substantially during exercise, however, particularly during the higher workloads. It is unlikely that this effect is attributable to diminishing activity of the drug since it is pharmacologically active for at least $\mathbf{1 5}$ minutes after sublingual administration (Goldstein et al., 1971). More probably, mechanisms reducing venous pooling during exercise in the upright position in the absence of drugs reduce the tendency to venous pooling initiated by GTN. Regardless of the exact mechanisms involved, our data indicate that the GTN-induced decrease in the left ventricular enddiastolic dimension observed at rest (Williams et al., 1965; Burggraf and Parker, 1974; DeMaria et al., 1974; Redwood et al., 1974) cannot be assumed to remain unaltered during exercise upright.

\section{LEFT VENTRICULAR DIMENSIONS IN} ANGINAL PATIENTS

Unlike the normal subjects, the patients with angina, as a group, showed a diversity in left ventricular dimensions at rest that probably reflected differences in the degree of disordered contraction of the region of myocardium traversed by ultrasound beam as well as differences in overall left ventricular performance (Fig. 4). Nevertheless, the pattern of response of the end-diastolic dimension in them was very similar to that of the normal subjects, a mean 9 per cent reduction in the end-diastolic dimension upon assuming the upright position being reversed at the lower level of exercise. The end-diastolic dimension again increased slightly at the higher level of exercise and, notably, at this point, just after the onset of angina, differed very little from the value during exercise before angina. In our study (all ambulatory patients) the production of angina by exercise in the upright position was never accompanied by a large abrupt expansion of left ventricular end-diastolic size. The effects of ischaemia were more clearly evident in the absence in nearly all of them of the normal decrease in the end-systolic dimension and rise in percentage shortening. This is consistent with the failure of the ejection fraction, measured by radionuclide cineangiography, to rise normally in patients with coronary disease performing exercise supine (Borer et al., 1977).

GTN, in doses producing a lowered blood pressure or an increased heart rate and a raised exercise capacity in every patient, caused changes in the end-diastolic dimension (Fig. 5, left) closely resembling those noted in the normal subjects. Thus, at rest in the upright position the enddiastolic dimension was substantially decreased relative to resting values after placebo. As in normal subjects, the decrease was less at higher exercise levels; the majority of patients showed very little decrease in end-diastolic dimension while exercising at intensities in excess of those necessary to produce angina in the absence of GTN. One such individual (Fig. 6) showed that a substantial increase in exercise capacity could occur with virtually no GTNinduced reduction in the simultaneously measured end-diastolic dimension. Thus, though certain patients during exercise after GTN have such a decrease, this is not invariable even when angina is prevented.

In most of the patients GTN had the same small and inconsistent effect on the end-systolic dimension and on percentage shortening as it had in the normal subjects. In two, both with relatively severe hypokinesia present on echocardiograms before GTN, however, the drug produced a large decrease in the 
end-systolic dimension and increase in percentage shortening during exercise. This finding must be regarded as inconclusive because of the small number but is consistent with the GTN-induced improvement in systolic function in hypokinetic segments recently shown by radionuclide cineangiography during exercise supine (Borer et al., 1978), and with earlier similar observations made at supine rest by contrast angiography (Helfant $e t$ al., 1974; Sniderman et al., 1974; McAnulty et al., 1975). The mechanism may be the alleviation of regional myocardial ischaemia resulting from a reduction in myocardial oxygen consumption, or an increase in coronary collateral flow to potentially ischaemic areas or, lastly, a reduction in left ventricular afterload having a disproportionately beneficial action on myocardium weakened by ischaemia (Theroux et al., 1976). Indeed, all three occur. It is worthy of note that improvement in systolic function was not always associated with a substantial reduction in the simultaneously measured end-diastolic dimension (Fig. 5, left) even when this was measured at the site of such improvement. Thus, neither objective benefit (the relief of systolic hypokinesia during exercise), nor subjective benefit (the prevention of angina) was consistently associated with a decrease in left ventricular end-diastolic dimensions.

IMPLICATIONS RELATING TO ANTIANGINAL EFFICACY OF GLYCERYL TRINITRATE

Our data have important implications in relation to possible mechanisms of GTN-induced relief of exertional angina in patients with chronic coronary occlusive disease. GTN has been thoughout to act, at least in part, by decreasing left ventricular dimensions during exercise. By diminishing the principal radii of curvature for each portion of the left ventricle (Burton, 1962), GTN could reduce left ventricular wall stress through the Laplace relation. Reduced wall stress during the first third of systole would lower myocardial oxygen consumption (Monroe, 1964) and thereby improve the balance of oxygen demand and supply. Reduced wall stress during diastole might also facilitate coronary blood flow to ischaemic areas by diminishing stretching forces on collateral vessels and capillaries. The Laplace relation, however, implies that wall stress would be reduced only in proportion to the decrease in left ventricular linear dimensions (such as the end-diastolic or end-systolic dimension) and not in proportion to the (much larger) associated decrease in left ventricular volume. Similar conclusions may be derived from calculations directly relating circumferential wall stress and left ventricular echocardiographic dimensions (Ratshin et al., 1974).
Our results favour the hypothesis postulating GTN-induced reduction in wall stress during exercise in the upright position in that the left ventricular end-diastolic dimension decreased to some degree in all 6 patients and substantially (over $10 \%$ ) for long periods in 2 of them.

In the other 4 patients, however, the observed decreases were very small (under $4 \%$ ). One patient (Fig. 6) had no measurable decrease during the time when, protected by GTN, he was not only free of angina but also exercising at a greater intensity than his previous maximum. It is possible that a very small decrease in left ventricular wall stress may lead to a disproportionately large fall in myocardial oxygen consumption but there is no experimental verification for this. Our data do not suggest that a decrease in left ventricular cavity size during early systole is an important mechanism by which GTN prevents or forestalls angina during exercise upright.

Although GTN-induced reductions in the enddiastolic dimension during exercise were small, associated decreases in left ventricular diastolic pressure during exercise may have been substantial (Parker et al., 1966). By decompressing collateral vessels and the capillaries within potentially ischaemic tissue, such decreases can significantly augment coronary collateral blood flow (Kjekshus, 1973). This influence, and the oxygen-sparing effect of a reduction in left ventricular systolic pressure, may be the most important benefits conferred by GTN, but our results suggest that, in certain patients, relief of localised systolic hypokinesia may also have a role. In addition to improving left ventricular systolic function, relief of hypokinesia might, in itself, exert a favourable influence by reducing local systolic wall stress, thereby reducing myocardial oxygen consumption specifically within potentially ischaemic portions of the left ventricle.

We wish to thank Dr Brian F. Robinson and Dr Aubrey Leatham of St. George's Hospital for their advice and encouragement.

\section{References}

Bevegård, B. S., and Shepherd, J. T. (1965). Changes in tone of limb veins during supine exercise. Fournal of Applied Physiology, 20, 1-8.

Borer, J. S., Bacharach, S. L., Green, M. V., Kent, K. M., Epstein, S. E., and Johnston, G. S. (1977). Real-time radionuclide cineangiography in the non-invasive evaluation of global and regional left ventricular function at rest and during exercise in patients with coronary artery disease. New England fournal of Medicine, 296, 839-844.

Borer, J. S., Bacharach, S. L., Green, M. V., Kent, K. M., Johnston, G. S., and Epstein, S. E. (1978). Effect of nitroglycerin on exercise induced abnormalities of left ventricular regional function and ejection fraction in 
coronary artery disease: assessment by radionuclide cineangiography in symptomatic and asymptomatic patients. Circulation, 57, 314-320.

Burggraf, G. W., and Parker, J. O. (1974). Left ventricular volume changes after amyl nitrite and nitroglycerin in man as measured by ultrasound. Circulation, 49, 136-143.

Burton, A. C. (1962). Physical principles of circulatory phenomena: the physical equilibria of the heart and blood vessels. In Handbook of Physiology, section 2: Circulation, Vol. 1, pp. 85-106. American Physiological Society. The Society, Washington, DC.

DeMaria, A. N., Vismara, L. A., Auditore, K., Amsterdam, E. A., Zelis, R., and Mason, D. T. (1974). Effects of nitroglycerin on left ventricular cavity size and cardiac performance determined by ultrasound in man. American fournal of Medicine, 57, 754-760.

Freyschuss, U. (1970). Cardiovascular adjustment to somatomotor activation: the elicitation of increments in heart rate, aortic pressure, and venomotor tone with the initiation of muscle contraction. Acta Physiologica Scandinavica, Suppl. 342, 5-63.

Goldstein, R. E., Rosing, D. R., Redwood, D. R., Beiser, G. D., and Epstein, S. E. (1971). Clinical and circulatory effects of isosorbide dinitrate: comparison with nitroglycerin. Circulation, 43, 629-640.

Helfant, R. H., Pine, R., Meister, S. G., Feldman, M. S., Trout, R. G., and Banka, V. S. (1974). Nitroglycerin to unmask reversible asynergy: correlation with postcoronary bypass ventriculography. Circulation, 50, 108-113.

Henry, W. L., Clark, C. E., and Epstein, S. E. (1973). Asymmetric septal hypertrophy. Circulation, 47, 225-233.

Kjekshus, J. K. (1973). Mechanism for flow distribution in normal and ischemic myocardium during increased ventricular preload in the dog. Circulation Research, 33, 489-499.

McAnulty, J. H., Hattenhauer, M. T., Rösch, J., Kloster, F. E., and Rahimtoola, S. H. (1975). Improvement in left ventricular wall motion following nitroglycerin. Circulation, 51, 140-145.

Monroe, R. G. (1964). Myocardial oxygen consumption during ventricular contraction and relaxation. Circulation Research, 14, 294-300.

Parker, J. O., Di Giorgi, S., and West, R. O. (1966). A hemodynamic study of acute coronary insufficiency precipitated by exercise with observations on the effects of nitroglycerin. American fournal of Cardiology, 17, 470-438.

Ratshin, R. A., Rackley, C. E., and Russell, R. O., jun (1974). Determination of left ventricular preload and afterload by quantitative echocardiography in man. Circulation Research, 34, 711-718.

Redwood, D. R., Henry, W. L., and Epstein, S. E. (1974). Evaluation of the ability of echocardiography to measure acute alterations in left ventricular volume. Circulation, 50, 901-904.

Redwood, D. R., Rosing, D. R., Goldstein, R. E., Beiser, G. D., and Epstein, S. E. (1971). Importance of the design of the exercise protocol in the evaluation of patients with angina pectoris. Circulation, 43, 618-628.

Sharma, B., Goodwin, J. F., Raphael, M. J., Steiner, R. E., Rainbow, R. G., and Taylor, S. H. (1976). Left ventricular angiography on exercise. A new method of assessing left ventricular function in ischemic heart disease. British Heart Fournal, 38, 59-70.

Sniderman, A. D., Herscovitch, P., Marpole, D., and Fallen, E. (1974). Restoration of regional wall motion by nitroglycerin therapy in patients with left ventricular asynergy. Chest, 66, 545-548.

Sonnenblick, E. H., Braunwald, E., Williams, J. F., jun, and Glick, G. (1965). The effects of exercise on myocardial force-velocity relations in intact, unanesthetized man; relative roles of changes in heart rate, sympathetic activity and ventricular dimensions. Fournal of Clinical Investigation, 44, 2051-2062.

Stein, R. A., Michielli, D., Fox, E., Sloan, M., and Krasnow, N. (1976). Continuous ventricular dimensions in man during supine bicycle exercise (abstract). Clinical Research, 24, 241.

Theroux, P., Ross, J., jun, Franklin, D., Kemper, W. S., and Sasayama, S. (1976). Regional myocardial function in the conscious dog during acute coronary occlusion and responses to morphine, propranolol, nitroglycerin, and lidocaine. Circulation, 53, 302-314.

Williams, J. R., jun, Glick, G., and Braunwald, E. (1965). Studies on cardiac dimensions in intact unanesthetized man. V. Effects of nitroglycerin. Circulation, 32, 767-771.

Requests for reprints to Dr Robert E. Goldstein, Building 10, Room 7B-15, National Institutes of Health, Bethesda, Maryland 20205, USA. 